\title{
Erratum
}

\section{Erratum to: Total pion-proton cross section from the new LHCf data on leading neutrons spectra}

\author{
R. A. Ryutin ${ }^{\mathrm{a}}$ \\ Institute for High Energy Physics, NRC “Kurchatov Institute”, Protvino 142 281, Russia
}

Published online: 8 December 2017

C) The Author(s) 2017. This article is an open access publication

Erratum to: Eur. Phys. J. C (2017) 77:114 https://doi.org/10.1140/epjc/s10052-017-4690-3

The LHCf data on $\mathrm{d} \sigma_{n} / \mathrm{d} E_{n}$ were considered in three rapidity ranges:

$\eta>10.76,8.99<\eta<9.22,8.81<\eta<8.99$.

On the page 3 of the article it was written, that we can use second and third regions, since $|t|$ values in these regions are of the order of $m_{\pi}^{2}$. This is not correct, since $|t| \sim 0.4 \rightarrow$ $1 \mathrm{GeV}^{2}$. In calculations of the Table 1 edge values of physical region in $|t|$ were used in the computer program instead of real $|t|$ values by mistake.

After more rigorous analysis resuls are following:

- we have to use the first region, where $\eta>10.76$ and real $|t|$ values are of the order of $m_{\pi}^{2}$;

- in the article Figs. 3, 6 and the Table 1 should be replaced by new ones (see below).

Additional errors in $\sigma_{\pi p}$ due to model dependance in the background estimations are not taken into account.

The original article can be found online at https://doi.org/10.1140/ epjc/s10052-017-4690-3.

a e-mail: Roman.Rioutine@cern.ch

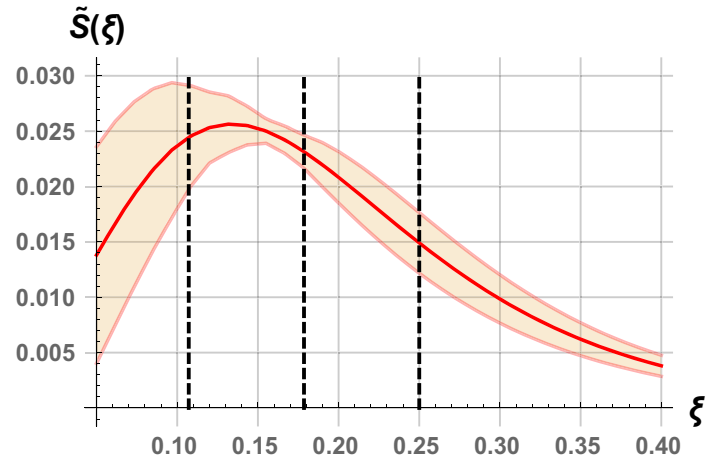

Fig. 3 Rescattering corrections multiplied by formfactors for $\sqrt{s}=$ $7 \mathrm{TeV}(\tilde{S}(s, \xi))$ integrated in the whole $t$ regions of the LHCf data [19]: $\eta>10.76$. Dotted vertical lines mark $\xi=0.107,0.179,0.25$, which are used to extract $\sigma_{\pi p}$ cross-sections

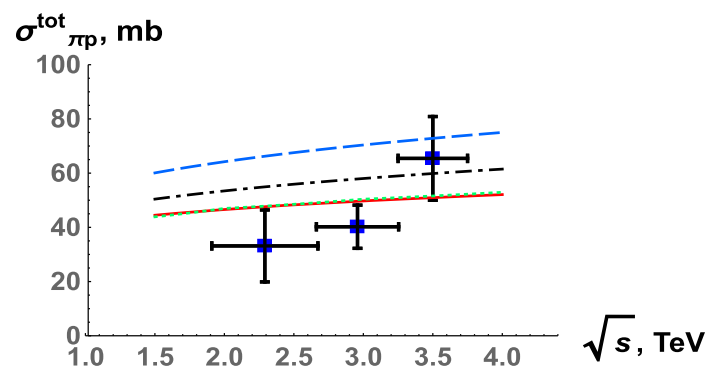

Fig. 6 Extracted total $\pi^{+} p$ cross-sections presented in the Table 1 versus different parametrizations: [34] (solid), [35] (dashed), [36] (dotted) and $[37,38]$ (dash-dotted). The interval of $t$ related to the $\eta$ region of the LHCf is $\eta>10.76$

Table 1 Values of the $\pi p$ total cross-sections extracted from the LHCf data [19] and also depicted in the Fig. 6. Corresponding average $|t|$ values and $q_{0}\left(q_{t}<q_{0}\right)$ are also shown. Backgrounds from $\rho$ and $a_{2}$ exchanges are taken into account

\begin{tabular}{llll}
\hline$\sqrt{s}, \mathrm{TeV}$ & $\sqrt{|t|} / m_{\pi}$ & $q_{0}, \mathrm{GeV}$ & $\sigma_{\pi p}^{\text {tot }}, \mathrm{mb}$ \\
\hline $2.291 \pm 0.382$ & $0.91 \pm 0.29$ & 0.132 & $33.15 \pm 13.1$ \\
$2.958 \pm 0.296$ & $1.41 \pm 0.166$ & 0.12 & $40.22 \pm 7.76$ \\
$3.5 \pm 0.25$ & $1.99 \pm 0.11$ & 0.112 & $65.43 \pm 15.15$ \\
\hline
\end{tabular}


Acknowledgements Thanks to Mikhail Ryskin for detailed comments to the article. The paper by KMR [Phys. Rev. D96 (2017) no.3, 034018; https://doi.org/10.1103/PhysRevD.96.034018 was also useful.

Open Access This article is distributed under the terms of the Creative Commons Attribution 4.0 International License (http://creativecomm ons.org/licenses/by/4.0/), which permits unrestricted use, distribution, and reproduction in any medium, provided you give appropriate credit to the original author(s) and the source, provide a link to the Creative Commons license, and indicate if changes were made.

Funded by SCOAP ${ }^{3}$. 